Carnets de géographes

GÉOGRAPHES

\title{
Entre mise à distance et proximité : la dimension spatiale du placement des mineurs dans le Rhône
}

\section{Fleur Guy}

\section{(2) OpenEdition}

1 Journals

Édition électronique

URL : http://journals.openedition.org/cdg/2138

DOI : $10.4000 /$ cdg. 2138

ISSN : 2107-7266

Éditeur

UMR 245 - CESSMA

Référence électronique

Fleur Guy, «Entre mise à distance et proximité : la dimension spatiale du placement des mineurs dans le Rhône », Carnets de géographes [En ligne], 3 | 2011, mis en ligne le 01 décembre 2011, consulté le 07 mai 2019. URL : http://journals.openedition.org/cdg/2138; DOI : 10.4000/cdg.2138

La revue Carnets de géographes est mise à disposition selon les termes de la Licence Creative Commons Attribution - Pas d'Utilisation Commerciale - Pas de Modification 4.0 International. 


\title{
ENTRE MISE À DISTANCE ET PROXIMITÉ : La dimension spatiale du placement des mineurs dans le Rhône
}

\author{
FLEUR GUY \\ Université de Lyon (Lumière Lyon 2) \\ UMR 5600/Bio-Géophile \\ Géographie \\ fleur.guy@univ-lyon2.fr
}

\begin{abstract}
Résumé
Intégré au dispositif de la Protection de l'Enfance et de la Protection Judiciaire de la Jeunesse, le placement en établissement correspond à la prise en charge, en dehors de son domicile familial, d'un mineur pour une durée donnée. Répondant à un objectif d'éloignement du jeune d'un milieu (familial et/ou social) considéré comme dangereux pour lui, cette prise en charge doit en même temps assurer la proximité entre le jeune et sa famille et l'accessibilité des structures nécessaires à son insertion. L'articulation de ces objectifs contradictoires implique de la part des acteurs sociaux une négociation avec l'espace, au travers notamment de mobilités quotidiennes. La mise en évidence de la dimension spatiale d'un dispositif d'action sociale permet ainsi d'interroger l'usage de l'espace par des acteurs sociaux.
\end{abstract}

\begin{abstract}
In France, institutional fostering is included within the Youth Protection and the Judiciary Youth Protection system. Institutional fostering is defined as follows: minor care, out of his/her domestic sphere, for a given period. This care is expected to meet a specific goal: get the child or teenager away from an environment (social and/or family) considered dangerous. On the other hand, though, that care needs to avoid a social isolation of that minor, both from an affective (family) and socio-professional (access to structures) point of view, since both dimensions are required for an effective social integration. As a consequence, social actors are confronted to contradictory goals; they try to overcome them by negotiating the space through daily mobilities. Hence, by underlining the spatial dimension of this social action plan, this article interrogates that very use of space by social actors.
\end{abstract}




\section{Introduction}

Alors que pour la majorité des enfants et des jeunes, les " espaces du quotidien " sont ceux du domicile familial, du quartier et de l'école, d'autres évoluent dans des environnements différents. Pour certains, cet espace du quotidien est celui de la rue (Tessier, 1995; Morelle, 2010), mais d'autres vivent, à l'inverse, dans des établissements plus ou moins fermés. En France, aux côtés de différentes mesures, le placement en établissement apparaît en effet comme une solution, plus ou moins temporaire, pour pallier aux difficultés que rencontrent certains mineurs. Cette mesure d'accueil se différencie des mesures dites " en milieu ouvert ", par lesquelles le mineur reste dans son milieu familial, et des mesures de placement familial. Plusieurs termes sont utilisés pour évoquer le placement en établissement. Les acteurs administratifs et associatifs parlent ainsi de " placement " mais aussi " d'accueil " ou "d'hébergement", termes auxquels s'ajoutent certains qualificatifs comme " hébergement d'urgence ", " accueil externalisé ", " placement pénal » ou encore "placement contenant ». Au-delà d'une diversité en termes de durée et de type de prise en charge, le placement sera ici envisagé comme le retrait d'un mineur de son milieu familial pour un temps donné et son accueil dans un établissement collectif, dans le double cadre institutionnel de l'Aide Sociale à l'Enfance (ASE) et de la Protection Judiciaire de la Jeunesse (PJJ).

Si la dimension spatiale de ce type de mesure apparaît évidente au regard de la terminologie employée par les acteurs sociaux (le " placement »), les géographes se sont peu intéressés à cet objet de recherche. Pourtant, l'espace géographique apparaît comme un enjeu majeur de cette prise en charge. Elle repose tout d'abord sur l'éloignement du mineur de son milieu familial et mobilise donc la distance géographique. Ce dispositif doit en même temps répondre à un impératif de proximité du jeune avec sa famille mais aussi avec la société en général. Le paradoxe entre la mise à distance et l'objectif d'insertion sociale, mis en évidence par Olivier Milhaud dans le cas des prisons françaises se retrouve donc ici (Milhaud, 2009). Les mineurs représentent cependant une population spécifique. L'incarcération concerne une faible part de la prise en charge des mineurs et le dispositif judiciaire est lié au dispositif administratif de la protection de l'enfance. La mesure de placement touche en effet deux catégories d'individus que les institutions tendent à séparer : d'un côté une enfance identifiée en danger dans son milieu familial, de l'autre une enfance perçue comme menaçante pour la société. Les mineurs constituent d'autre part une population spécifique car considérée comme "en devenir ». La question des apprentissages sociaux et de la scolarisation se pose ainsi de manière différente par rapport à une population adulte. Enfin, la définition juridique d'un individu mineur pose la question de sa responsabilité civile et donne une place importante à la famille dans le cadre de la prise en charge. Si les spécificités de la population mineure semblent jouer en faveur d'une prise en charge axée sur la proximité avec la famille et l'environnement social (école, activités de loisirs), le " placement » ne constitue-t-il pas dans le même temps un processus de mise à distance ? En quoi la gestion de ce paradoxe implique-t-elle de la part des acteurs concernés une "négociation avec l'espace ", en lien avec la catégorisation des publics ? 
Le concept de mobilité permet également d'aborder le placement dans sa dimension spatiale. Les mobilités quotidiennes à l'extérieur de l'établissement, qu'elles soient autorisées ou non (fugues), permettent de comprendre les différentes gestions de la distance géographique dans le cadre du placement. La mobilité, valeur montante de la société (Bacqué, Fol, 2007) et présentée comme une compétence nécessaire à I'insertion sociale, notamment des jeunes (Gachelin, 2007), peut également être perçue négativement lorsqu'elle entrave les dispositifs de contrôle et de surveillance. Le concept de mobilité est également mobilisé ici à une plus large échelle temporelle, celle de la vie des mineurs, en considérant le placement comme une mobilité résidentielle. Dans ce cas, les conséquences sociales de ce type de mobilité spatiale sont envisagées.

Le "placement " questionne en effet les relations entre la position sociale des individus et leur localisation dans l'espace géographique, s'intégrant ainsi dans un débat géographique plus large. Le concept de "place", qui renvoie à la fois à la localisation dans l'espace géographique mais aussi à la place dans la société (Cresswell, 1996), revêt alors une portée heuristique puisqu'il permet d'appréhender l'espace comme dimension de la société et de comprendre comment " les rapports à l'espace participent de la définition des positions sociales " (Ripoll, Tissot, $2010: 6$ ). L'espace n'est pas un facteur en soi agissant sur le social, ou un simple cadre de l'action, mais une dimension des processus sociaux (Veschambre, 2006). Si la territorialisation croissante des politiques publiques et la "spatialisation des problèmes sociaux" (Poupeau, Tissot, 2005) ont invité les chercheurs à questionner les relations entre territoire et action sociale (Bouquet, Madelin, Nivolle, 2007 ; lon, 2005), le concept de territoire renvoie essentiellement au territoire vécu ou institutionnel. Pourtant, une prise en compte globale de l'espace, considéré plus seulement comme espace vécu ou délimité politiquement, mais comme dimension du social, montre que les acteurs sociaux sont amenés à "faire avec l'espace " dans l'ensemble de leurs pratiques (Lussault, Stock, 2010 : 15). Ce "faire avec l'espace » peut selon les situations et les acteurs être une ressource ou une contrainte puisque :

"les " acteurs " sociaux font avec la distance, les cadres matériels et les contextes sociaux, au double sens du terme : ils ne peuvent pas ne pas y être confrontés, et ils en usent ou cherchent à le faire, mais dans la mesure de leurs moyens » (Ripoll, 2006 : 209).

Le cas du placement de mineurs interroge la manière dont les acteurs sociaux se saisissent de l'espace géographique, en l'intégrant au cœur de la prise en charge des mineurs, en même temps qu'il invite à envisager les effets de ce placement sur les représentations des acteurs sociaux, et sur la catégorisation des jeunes pris en charge. Cet article interroge la prise en charge des mineurs via un placement en établissement et s'appuie sur une étude bibliographique, des entretiens semi-directifs (avec les présidents de trois associations gérant des établissements dans le Rhône, avec les directeurs et directrices de certains d'entre eux, situés en milieu urbain, périurbain et rural et avec les représentants des services du Conseil général, de la PJJ et de l'administration pénitentiaire) et la participation aux présentations collectives de certains établissements (dans le cas de l'Etablissement Pénitentiaire pour Mineurs (EPM), Meyzieu et de la Maison d'Enfants à caractère Social (MECS) "Les Glycines ", Lyon). 


\section{Protéger et/ou punir le mineur, le placement dans son contexte institutionnel et théorique}

Les recherches actuelles sur l'enfance tendent à l'appréhender comme un phénomène plus social que biologique (Holloway, Valentine, 2000). L' " enfance », tout comme la " jeunesse ", renvoie à la constitution d'un groupe doté d'intérêts communs rapportés à un âge biologiquement défini (Bourdieu, 1980). Le terme de " mineur " fait quant à lui référence à un statut juridique, rapporté à une limite d'âge biologique, et désigne des individus qui ne bénéficient pas d'une pleine capacité d'exercice civil et de responsabilité pénale. Les dispositifs que nous abordons renvoient à la fois à "l'enfance " (Aide Sociale à l'Enfance) et à la " jeunesse " (Protection Judiciaire de la Jeunesse). L'utilisation différenciée des termes ne renvoie pas ici spécifiquement à une limite d'âge biologique, puisqu'un individu du même âge peut être pris en charge par l'un ou l'autre des dispositifs, mais plus à des attributs sociaux, avec une opposition entre enfance en danger et jeunesse délinquante mettant en danger la société. Le placement de mineurs en établissement entre en effet dans le cadre de deux types de procédures différenciées, s'intégrant à un dispositif institutionnel divisé en deux pôles, celui de l'ASE et celui de la PJJ. Cette catégorisation des publics s'appuie également sur le sexe des jeunes accueillis. En effet, la grande majorité des établissements est aujourd'hui mixte mais la prise en charge des mineurs des deux sexes est souvent séparée, avec des unités de vie distinctes ou, dans de rares cas, des établissements non mixtes. La question du genre dans le choix de l'établissement, comme dans le déroulement de la prise en charge, mériterait ainsi d'être étudiée. Si les procédures de I'ASE et de la PJJ tendent à être de plus en plus distinctes dans le contexte législatif actuel, elles ont néanmoins des origines communes et se rejoignent dans certaines pratiques. La dimension spatiale de ces pratiques, mise en relation avec la catégorisation croissante des publics, mérite ainsi d'être interrogée.

\section{"Mineurs en danger »/" mineurs délinquants " : une prise en charge différenciée}

Le placement en établissement s'inscrit dans un dispositif institutionnel complexe. La distinction entre protection du mineur en danger et sanction du mineur délinquant ne suffit pas à rendre compte de cette complexité puisqu'elle se double notamment d'une dichotomie entre mesure administrative et judicaire. On peut ainsi différencier trois types de mesures, administrative, judiciaire civile et judiciaire pénale, faisant intervenir de multiples acteurs, dans le processus de décision de la mesure autant que dans son application. Alors que l'autorité administrative intervient seulement au titre de la protection de l'enfance, l'autorité judicaire est compétente pour les deux domaines. Suite aux lois de décentralisation du début des années 1980, le département est l'échelon territorial privilégié de la protection de l'enfance. Le président du Conseil Général, au travers du service de l'ASE, constitue ainsi le "centre de gravité du dispositif ", assurant notamment les fonctions de recueil de données, d'évaluation et de traitement des situations (Borgetto, Lafore, 2009: 28"). Le président doit cependant coopérer dans certains cas avec l'autorité judiciaire. En effet, la protection de l'enfance s'est constituée autour d'enfants dont le milieu familial était considéré 
comme défaillant ou dangereux par la puissance publique et dont il fallait retirer l'enfant. Cette procédure, qui perdure aujourd'hui avec le placement en famille d'accueil ou en établissement, porte atteinte aux droits de l'autorité parentale et nécessite donc dans certains cas une intervention judiciaire. Les évolutions législatives récentes tendent à amoindrir le rôle de l'autorité judiciaire dans la protection de l'enfance au profit de l'autorité administrative ${ }^{1}$. L'affaiblissement du rôle du juge pour enfants dans les mesures relevant de l'ASE contribue à une séparation croissante entre les prises en charge des mineurs en danger et des mineurs délinquants.

La prise en charge des mineurs ayant commis des infractions est intégrée à la sphère pénale. C'est alors le juge des enfants qui est au centre du dispositif, assisté des services de la PJJ. Cette prise en charge se fonde du point de vue législatif sur l'ordonnance du 2 février 1945 qui instaure le principe d'une juridiction spécifique aux mineurs, privilégiant la visée éducative des interventions judiciaires. Adoptée le même jour que la loi $n^{\circ}$ 2007-29" du 5 mars 2007 réformant la protection de l'enfance, la loi $\mathrm{n}^{\circ}$ 2007-297 relative à la prévention de la délinquance institue un «jeu de différenciation des territoires, en fonction des cibles de l'action publique, enfance en danger ou enfant délinquant " (Choufri, 2010 : 44) en plaçant notamment le maire et l'échelon communal comme "pivot de la prévention de la délinquance " (Choufri, 2010 : 46). Les reconfigurations institutionnelles issues des modifications législatives récentes vont donc dans le sens d'une segmentation de la prise en charge des mineurs en danger et des mineurs ayant commis des infractions. D'un côté la subsidiarité des mesures judiciaires sur les mesures administratives dans le cadre de la protection de l'enfance confirme le rôle central du Conseil Général dans ce domaine. L'action du juge des enfants et de la PJJ est quant à elle recentrée sur les mesures pénales. Ces recompositions s'accompagnent d'évolutions territoriales. En effet, les actions de prévention de la délinquance se recentrent à l'échelle de la commune. L'échelon départemental est quant à lui privilégié dans la prévention, le repérage et la prise en charge de l'enfance en danger, tandis que la prise en charge des mineurs au pénal s'effectue à un échelon déconcentré interdépartemental.

\section{"On ne peut pas découper un individu en rondelles »}

Les prises en charge des mineurs en danger et des mineurs ayant commis des infractions apparaissent pourtant comme des domaines fortement liés, notamment au vu de l'histoire des dispositifs législatifs relatifs à l'enfance en danger. Depuis la Révolution française, les deux objectifs, l'intérêt de l'enfant et celui de la société, semblent se confondre. Les mesures prises dès les lendemains de la Révolution française et le rôle croissant de l'Etat dans la prise en charge de l'enfance sont ainsi d'abord liés à des raisons politiques. Il s'agit de " renouveler de fond en comble les enfants sans famille, mal élevés, socialement défectueux ", pour qu'ils incarnent les valeurs et idéaux de la nouvelle nation et deviennent des citoyens à part entière

\footnotetext{
${ }^{1}$ La loi n`2007-293 du 5 mars 2007 réformant la protection de l'enfance rééquilibre les compétences entre autorité administrative (Conseil Général) et judiciaire (Juge des enfants), au profit de l'autorité administrative. Alors qu'il possédait un rôle de pivot dans le système antérieur, le juge n'intervient plus que de manière subsidiaire, après échec d'une mesure décidée par le président du Conseil Général, ou en cas de refus de coopération des parents.
} 
(Jablonka, 2010 : 16). Au XIX ème siècle, la volonté de protéger l'enfant trouve son origine dans des " considérations initiales d'ordre public " (Pédron, 2005 : 21). Pierre Pédron montre l'ambivalence du concept de " protection judicaire ", qui vise autant la protection de l'enfance que la protection de la société. Pour répondre à la problématique sociale des mineurs délinquants, les pouvoirs publics mettent en place des mesures principalement éducatives, mais qui ont aussi pour vocation de protéger la société. L'«Education Surveillée ", l'institution que remplacera la PJJ à partir de 1990, montre bien ce double objectif d'éducation et de contrôle. Cette ambiguïté se retrouve dans l'usage qui est fait du dispositif législatif. Des mesures pénales peuvent ainsi être utilisées comme un moyen d'intervenir sur les enfants en situation de danger. Le rôle central du juge des enfants dans le dispositif de protection de l'enfance, avant la réforme issue de la loi de 2007, lui permettait ainsi de " jouer sur les registres civils et pénaux en fonction de la situation du jeune et de sa famille " (Borgetto, Lafore, 2009 : 296).

En effet, si la distinction entre une enfance victime et une enfance coupable se justifie au regard de la loi, les acteurs sociaux sont conscients de la porosité des deux publics. $S^{\prime}$ « il n'y a pas de différence radicale » entre « les profils des mômes placés au pénal et au civil » (Directeur d'association, 30/11/2010), nos interlocuteurs précisent toutefois que tous les enfants faisant l'objet d'une mesure administrative ne sont pas des délinquants potentiels. Ils soulignent à l'inverse la présence constante de difficultés familiales chez les jeunes délinquants. Ce constat est présent dès le début du $X X^{\text {ème }}$ siècle, où la cause principale de la délinquance des mineurs est identifiée dans leur milieu familial (Bourquin, 2007). Cette dialectique entre " enfance victime " et " enfance coupable » se retrouve aujourd'hui. En 2002, le "Rapport Schosteck ", met ainsi en évidence les relations entre "violences subies-violences agies " chez les populations jeunes, et "l'étroite imbrication des concepts de mineurs auteurs et de mineurs victimes" (2002: "9). Si, comme le souligne une représentante du Conseil Général du Rhône, " un individu ne peut pas être découpé en rondelles, une partie à protéger, l'autre à punir ", les dispositifs actuels impliquent une catégorisation croissante des publics. Bien que rattachées à deux institutions différentes, les prises en charge opèrent parfois avec les mêmes moyens. Le placement en établissement est ainsi prononcé à la fois au titre de la protection administrative et de la protection judiciaire civile et pénale.

\section{Placement et catégorisation spatiale}

Le département du Rhône propose une palette importante d'établissements. En effet, si on peut parler d'une mesure, "le placement", les caractéristiques des établissements peuvent être très différentes, à la fois selon leur type, et au sein d'un même type d'établissements en fonction du "projet d'établissement ". Ils sont en majorité gérés par le secteur associatif et sont habilités, soit par le Conseil Général, soit par la PJJ, selon qu'ils accueillent des jeunes en protection administrative, judiciaire civile, ou dans le cadre de poursuites pénales. Certains établissements ne peuvent accueillir des mineurs qu'au titre pénal mais la plupart d'entre eux possèdent une "double habilitation ». Celle-ci est fréquente dans le cas des MECS puisque plus de deux-tiers des MECS du Rhône peuvent accueillir des jeunes au titre de l'ASE ou 
$d^{\prime}$ un placement judiciaire ${ }^{2}$. Cette souplesse du cahier des charges des établissements doit cependant être relativisée au regard des pratiques.

Plusieurs auteurs mettent en effet en évidence les différentes logiques, parfois contradictoires, qui entrent en jeu dans la décision de placement. La cohérence entre le cahier des charges, le projet éducatif de l'établissement et les besoins du mineur est parfois subordonnée à de multiples logiques institutionnelles et notamment aux négociations entre les principaux acteurs cette mesure (Jamet, 2010 ; Sicot, Maigne, 2005). En outre, si la double habilitation est majoritaire, l'accueil des mineurs en danger et des mineurs ayant commis des infractions se fait de manière différenciée, certaines structures refusant d'accueillir les jeunes en placement pénal ou sortant de certains établissements.

Selon le rapport d'évaluation sur "l'insertion des adolescents en difficulté » de 199", la multiplicité des filières représente un atout dans la gestion des publics mais ne favorise pas l'insertion des jeunes puisque "les filières apparaissent univoques: on peut " monter " vers le pénal, rarement " descendre » et retrouver le terrain familial et social » (Comité interministériel de l'évaluation des politiques publiques, 199" : 90). $\mathrm{Ce}$ constat est à mettre en relation avec "l'effet-miroir" entre l'identité de l'institution et celle des mineurs puisque "le degré de coercition d'un lieu de placement détermine dans l'esprit des professionnels l'ancrage dans la délinquance des mineurs accueillis " (Jamet, 2010 : 40). La stigmatisation des mineurs est ainsi redoublée par le lieu même de leur placement. Les " effets de lieu » décrits par Pierre Bourdieu principalement à l'échelle du quartier et la "dégradation symbolique " réciproque entre le quartier et ses habitants se retrouvent ici à une échelle plus fine (Bourdieu : 167). Cette assignation identitaire apparaît dans le discours des acteurs qui parlent de " jeunes de CER (Centre Educatif Renforcé) » ou de " jeunes d'EPM » tout en dénonçant cette stigmatisation qui accroît les difficultés d'insertion des mineurs placés. Le fait même, pour un jeune, d'avoir été accueilli dans un nombre important d'établissements est négatif aux yeux des acteurs sociaux. La mobilité est alors associée à une difficulté d'adaptation du jeune, à son incapacité à se soumettre aux règles de vie en collectivité.

\section{Le « placement » : entre mise à distance et proximité}

\section{L'éloignement comme protection de l'enfant et de la société}

Les dispositifs actuels de la protection de l'enfance et de la jeunesse trouvent leur origine dans les dispositions législatives prises à la fin du XVIII ${ }^{\mathrm{èm}}$ siècle en faveur d'une éducation publique. Cette période correspond à l'essor de la scolarisation en internats, fondée sur la « réclusion de l'enfant et du jeune loin du monde et de sa propre famille [...] considérée comme l'une des formes idéales de l'éducation " (Ariès, 1960 : “ 1“). Touchant l'ensemble de la société, les réflexions sur le pouvoir émancipateur de I'instruction se développent également en réaction à une autorité paternelle considérée comme défaillante. La prise en charge des mineurs dans des établissements

\footnotetext{
${ }^{2}$ Fichier National des Etablissements Sanitaires et Sociaux : http://finess.sante.gouv.fr/index.jsp
} 
est alors rendue possible par le transfert des compétences en matière d'éducation de la puissance paternelle vers la puissance d'Etat. Certains dispositifs d'éducation collective voient ainsi le jour en amputant le droit des familles et les établissements créés à cette époque reposent donc sur un nécessaire éloignement du milieu familial des jeunes, ainsi que sur les vertus de l'environnement rural dans le processus de réhabilitation de l'enfant « inadapté ».

Autant que la famille, c'est en effet le « milieu » dans lequel a grandi l'enfant qui est rendu responsable de ses vices. L'aliénisme, à la fin du XIX ${ }^{\text {ème }}$ siècle, théorise la transmission héréditaire des vices mais aussi la transmission de dégénérescences produites par le milieu. Le milieu mis en cause est social mais possède aussi une composante spatiale forte : il s'agit du milieu urbain en pleine expansion suite à la révolution industrielle. Les thèses soutenues par le courant aliéniste justifient donc un déracinement du jeune de son milieu d'origine : si un milieu est responsable de l'inadaptation sociale des enfants et des jeunes, leur déplacement dans un autre milieu permettra leur réhabilitation dans la société. En réaction au développement du vagabondage en lien avec l'industrialisation et le développement urbain, les vertus salvatrices de la terre et de la campagne sont prônées (Pédron, 2005). Le caractère pathogène du milieu urbain mis en dialectique avec un milieu rural sain apparaît dans l'argumentation des défenseurs de la colonie agricole au XIX ${ }^{\text {ème }}$ siècle :

"On commence à reconnaître aujourd'hui les services que ces divers établissements rendent à la société en formant pour l'agriculture des sujets instruits; en domptant par le travail, la discipline, et une éducation morale et religieuse, des natures perverties et dégénérées, en fortifiant des tempéraments usés, débiles, souffreteux, et préparant au pays une génération vigoureuse et intelligente, destinée à peupler non pas nos manufactures qui regorgent de bras, et dans lesquelles la vie d'agglomération est aussi délétère pour l'âme que pour le corps, mais à repeupler nos campagnes, si malheureusement délaissées » (Dugat, Lamarque, $1850:$ 5-6).

Le recours à la colonie agricole, soutenu par les sciences psychiatriques et pénitentiaires, repose bien sur cette idée d'une " régénération à la française, fondée sur une rupture avec le milieu d'origine " et le mythe agraire de la métamorphose (Jablonka, $2010: 12{ }^{\prime \prime}$ ). II s'inscrit en outre dans un contexte d'exode rural et d'un besoin de main d'œuvre en milieu agricole. Bien que remis en cause par l'échec des colonies agricoles, l'intérêt du milieu rural pour la prise en charge de jeunes en difficulté reste toujours d'actualité. La majorité des établissements de placement du Rhône se trouve hors des "villes centres" des unités urbaines. En effet, sur 85 établissements se trouvant dans des unités urbaines, seulement 19 sont localisés dans des communes centre de ces unités urbaines. Certains établissements sont en outre situés dans des communes rurales (Figure 1). Cette localisation est en partie un héritage du XIXème siècle, comme le souligne un directeur d'association: "Les établissements ont été construits à distance, en opposition avec le lieu de vie, le milieu naturel des gamins. " (Directeur d'association, 30/11/2010). 
Figure 1 : Localisation des établissements de placement dans le Rhône

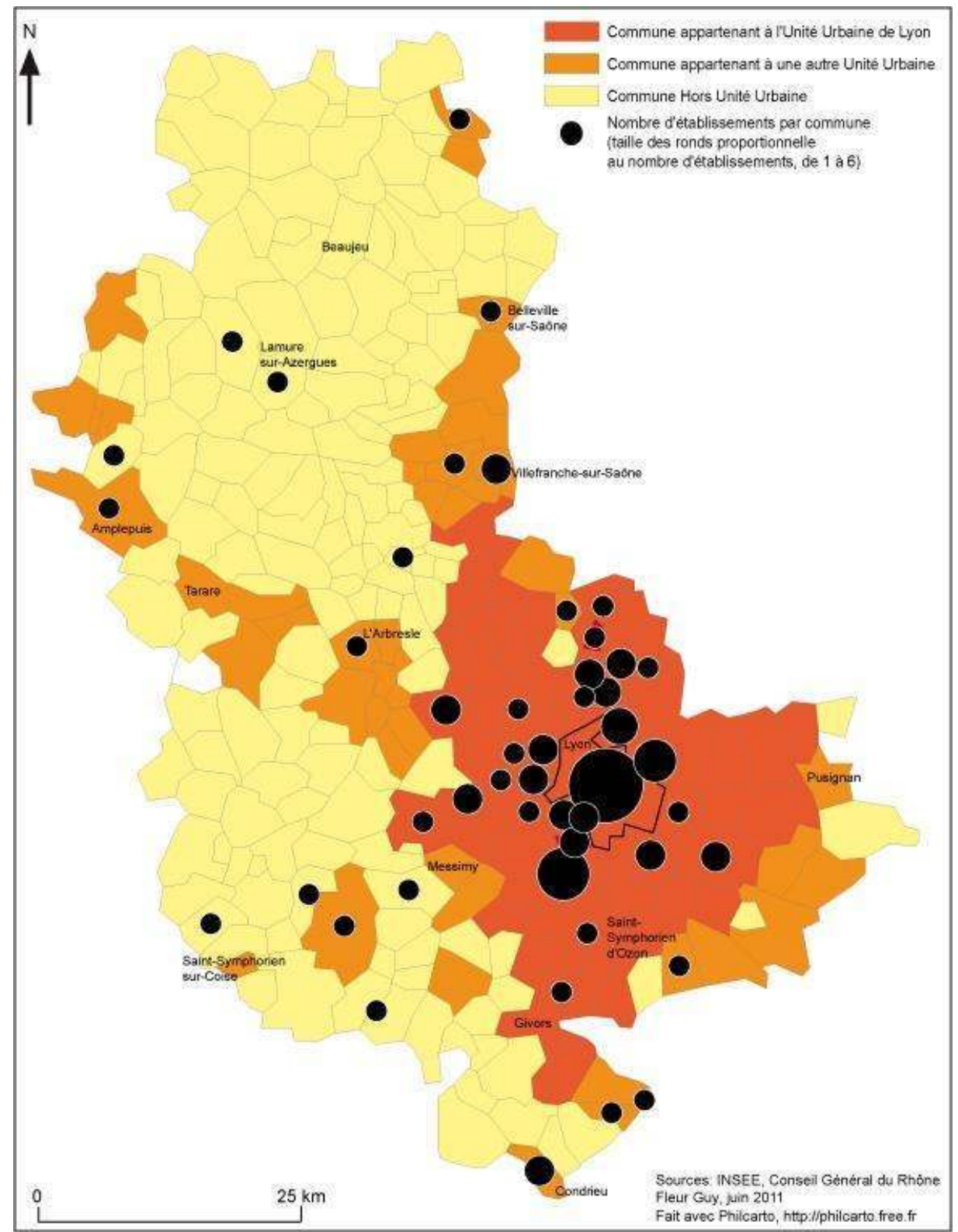

D'autres facteurs expliquent aussi leur localisation. La MECS Les Pierres dorées, située dans la commune rurale de Frontenas (Rhône), occupe ainsi un ancien cuvage, racheté en $19{ }^{\prime \prime} 0$ par des patronnesses lyonnaises. Si le projet est bien alors de permettre à des enfants lyonnais de passer des vacances à la campagne, la disponibilité de locaux agricoles joue dans le choix de la localisation. La présence toujours importante d'établissements en milieu rural peut également s'expliquer par l'intérêt qu'y trouvent certains élus locaux (plus d'enfants scolarisés sur leur commune, emplois liés aux établissements, etc.).

Les vertus du milieu rural sont encore aujourd'hui mises en avant pour ce type de prise en charge. La présentation d'une MECS du Rhône sur son site internet insiste par 
exemple sur la situation de l'établissement « au milieu des vignes beaujolaises et des fameux cerisiers " et la participation des enfants aux " activités locales telles que les vendanges, la cueillette des cerises, les manifestations sportives et culturelles... " . Les mêmes arguments sont repris dans le cas du placement pénal, avec par exemple le premier Centre Educatif Fermé (CEF), ouvert en mars 200" à Lusigny (Allier), dont les vertus naturellement pédagogiques et apaisantes du cadre sont vantées à plusieurs reprises (Mucchielli, 2005). La nécessité d'une rupture avec le milieu d'origine du jeune est inscrite dans le cahier des charges des Centre Educatifs Renforcés (CER) et reprise dans les projets d'établissements et le discours des acteurs sociaux:

"Dans les CER, on prend en charge des enfants délinquants qui se coupent de l'adulte, remettent en cause son autorité. Le principe est de brouiller les repères, rompre avec le milieu pour mieux se réengager. Le CER "Rang'donné " est centré sur l'équitation. Le séjour est marqué par une rupture à l'étranger qui permet un reconditionnement physique parce que ces jeunes ne dorment plus, ils fument... Au Maroc ils font aussi un chantier humanitaire à 900 kilomètres au sud. On les dépayse complètement, ils prennent de la distance, ils éprouvent le changement physique. C'est des mômes qu'il faut repositionner, qu'il faut sortir d'une trajectoire délinquante." (Directeur d'association, 30/11/2010).

Le rapport à l'espace est ici central dans ce qui apparaît comme une véritable transformation du jeune. Comme au cours des siècles précédents, cette rupture est présentée comme nécessaire dans la mesure où l'environnement du jeune est en partie considéré comme responsable de ses problèmes. C'est désormais le "quartier » qui est perçu comme un espace criminogène. Le "quartier", comme catégorie d'action publique, s'est en effet construit sur l'idée d'un milieu criminogène et pathogène. Dès les années 1960, la critique urbanistique et architecturale des grands ensembles s'accompagne d'une dénonciation des conséquences du cadre bâti sur la vie sociale et psychologique des habitants, et notamment sur " les jeunes » (Tissot, 2007).

L'éloignement reste un moyen de protéger le jeune de sa famille ou de le mettre à l'écart d'un environnement perçu comme pathogène. Cette mise à distance peut aussi être mise en relation avec l'autre objectif de la prise en charge, celui de protection de la société. Déjà avec l'ordonnance de 1945, qui crée l'Education surveillée et instaure la primauté de l'éducation sur la sanction dans la prise en charge des jeunes délinquants, le "placement lointain » ne répond pas seulement à un objectif d'éducation et de réadaptation sociale. Touchant des enfants dont les professionnels ont du mal à déterminer s'ils sont victimes ou coupables, il relève également de la "dette à payer à la société » (Bourquin, 2007 : 9). Des études montrent ainsi que le placement constitue une réponse "à la demande de sécurité, de tranquillité d'un quartier qui subit les délits d'un jeune» (Sicot, Maigne, 2005 : 99). On trouve la même logique dans les opérations "Prévention Eté » ou "Anti Eté-chaud », mises en place au début des années 1980 suite aux « rodéos » dans le quartier des Minguettes (LyonVénissieux). Elles avaient ainsi « pour objectifs premiers d'éloigner les jeunes les plus turbulents des cités et, donc, de ramener le calme " (Lapeyronnie, 200" : 4). Les exigences d'ordre public auxquelles répondent certains types de placement dits 
" contenants" les rapprochent ainsi de l'institution carcérale dans son objectif de régulation et de gestion de certaines populations. Le "rôle extra-pénologique du système pénal comme instrument de gestion des groupes dépossédés et déshonorés " est ici présent dans le cas d'un dispositif de politique sociale et invite à replacer l'institution carcérale " au sein du système complet des stratégies visant à réguler les pauvres, dont les politiques sociales » (Wacquant, 2001 : " 2).

L'éloignement du mineur, de sa famille d'une part, de son quartier d'autre part, reste aujourd'hui une dimension centrale du placement. II résulte non seulement de la localisation des établissements, héritée du XIXème siècle, mais aussi d'un double objectif de protection du mineur et de la société. Cette mise à distance est cependant aujourd'hui nuancée par un impératif de " proximité ».

\section{La proximité, un impératif relatif ?}

La nécessité pour l'enfant de conserver un lien avec sa famille est affirmée dans plusieurs textes législatifs. Elle apparaît dans la Convention Internationale des Droits de l'Enfant et dans ses transpositions dans la législation française. La protection de l'enfance s'articule en effet autour de deux objectifs : " préserver autant que faire se peut les liens entre l'enfant et ses parents et promouvoir le milieu familial de façon à ce qu'il puisse, à terme, assumer ses obligations éducatives selon les normes sociales en vigueur " (Borgetto, Lafore, 2009 : 291). L'impératif de conservation des liens avec la famille, s'il est parfois remis en cause par le trop grand danger que représente un contact avec les parents pour l'enfant, est largement pris en compte par les acteurs sociaux de ce domaine, qui font état de l'apparition récente de cette priorité. "C'est pas comme avant. La loi de 2002 a vraiment permis une réflexion sur la proximité. Avant le gamin pouvait se retrouver à perpette " (Directrice d'une MECS, 04/01/2011).

"Le placement c'est un traumatisme, c'est une rupture, on doit prendre en compte la place des parents. La prise en compte de la dynamique familiale est essentielle, je dis ça parce que dans les années 70 , on était les sauveurs, la famille était incapable. On était dans la substitution, maintenant on est dans la suppléance, et même la co-éducation. " (Directeur d'association, 06/02/2011)

Cette évolution dans le rapport des institutions de la protection de l'enfance et de la PJJ aux familles et la nécessité de conserver les liens familiaux du mineur placé impliquent une réflexion sur la localisation de l'établissement choisi pour le placement. Une distance physique importante du domicile, mais aussi un manque d'accessibilité, notamment en transports en commun, sont en effet des freins au maintien des liens familiaux. Là encore, les acteurs sociaux rencontrés soulignent le caractère récent de ces préoccupations:

"C'est sûr que la notion de territoire est beaucoup plus présente dans la tête des directeurs d'établissements qu'il y a vingt ans où c'était pas du tout un problème d'envoyer les gamins à soixante-dix kilomètres du domicile familial. " (Directeur d'association, 05/01/2011) 
Or l'offre d'établissements habilités est déséquilibrée dans le Rhône, avec une différence importante entre le nombre d'enfants nécessitant un placement et le nombre de placés à l'échelle des cantons (Figure 2).

Figure 2 : Le placement : un déséquilibre entre les besoins et l'offre dans les cantons du Rhône

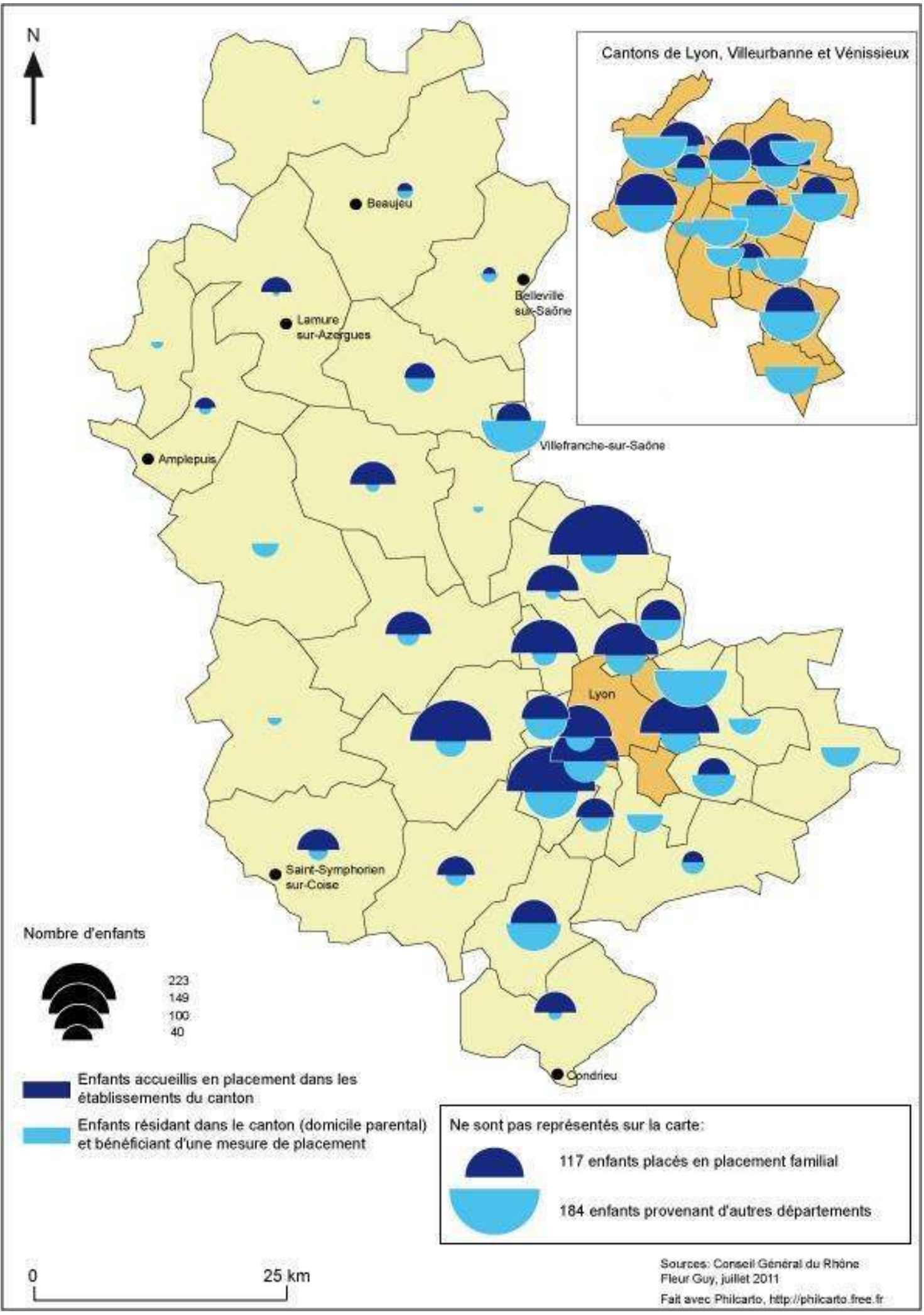


Cette carte s'appuie sur l'adresse administrative des établissements qui possèdent parfois des annexes dans d'autres cantons. Malgré cette approximation liée à la disponibilité des données, elle montre bien un déséquilibre qui s'explique par les différences de taux de population dans les cantons et qui ne permet pas toujours de respecter le principe de proximité avec le domicile familial. La réponse d'un directeur d'association, interrogé sur la contrainte que peut représenter la localisation de certains établissements, montre la difficulté d'adaptation à ces localisations : "On bricole. On fait du bricolage sur la base de structures figées " (Directeur d'association, -0/11/2010). Cette distance entre l'établissement et le lieu de vie des parents pose par exemple problème pour organiser les rencontres entre les parents et les enfants.

La localisation des établissements doit également prendre en compte leur proximité avec les services et ressources mobilisés dans le cadre de la prise en charge, comme les établissements scolaires ou médicaux. Le site internet du CER «Rang'donné » de Lentilly (Rhône) montre bien l'articulation des objectifs de mise à distance et de proximité: "Le site présente l'intérêt d'un lieu à la fois décalé et proche de l'agglomération lyonnaise, apportant un dépaysement et une rupture, en fournissant par ailleurs un accès facile aux prestataires économiques, pédagogiques, éducatifs ou sanitaires " ${ }^{4}$. Si la proximité, envisagée à la fois en termes de lien aux familles et de lien au territoire, est prise en compte de manière croissante par les différents acteurs, elle est essentiellement appréhendée en termes de distance physique et d'accessibilité via les transports en commun. Cette proximité peut également être envisagée à une échelle plus fine, en questionnant notamment le rapport des établissements et des individus qu'ils hébergent à l'environnement extérieur.

Ainsi, la distance physique n'est pas le seul facteur invitant à relativiser la proximité. En effet, comme le montre O. Milhaud dans son étude sur les prisons françaises, la proximité spatiale " n'empêche pas une discontinuité radicale entre le dedans et le dehors» (2009: 80). L'opposition entre un "dedans", correspondant à l'établissement et les services qui y sont proposés, et un " dehors " est constante dans le discours de ces acteurs. Lorsqu'ils évoquent ce type de prise en charge, les acteurs disent ainsi faire "du dedans-dehors " (Directrice de MECS, 09/02/2011) ou jouer sur la « relation intérieur/extérieur. " (Directeur d'association, 05/01/2011). La prise en charge joue donc elle-même sur cette dialectique du " dedans " et du " dehors ", qui peut être matérialisée par des murs d'enceinte marquant la rupture dans l'espace (Figures" et " bis).

\footnotetext{
${ }^{4}$ http://www.slea.asso.fr/cer/lieux.html
} 
Figure 3 et 3 bis : Une MECS: un espace ouvert

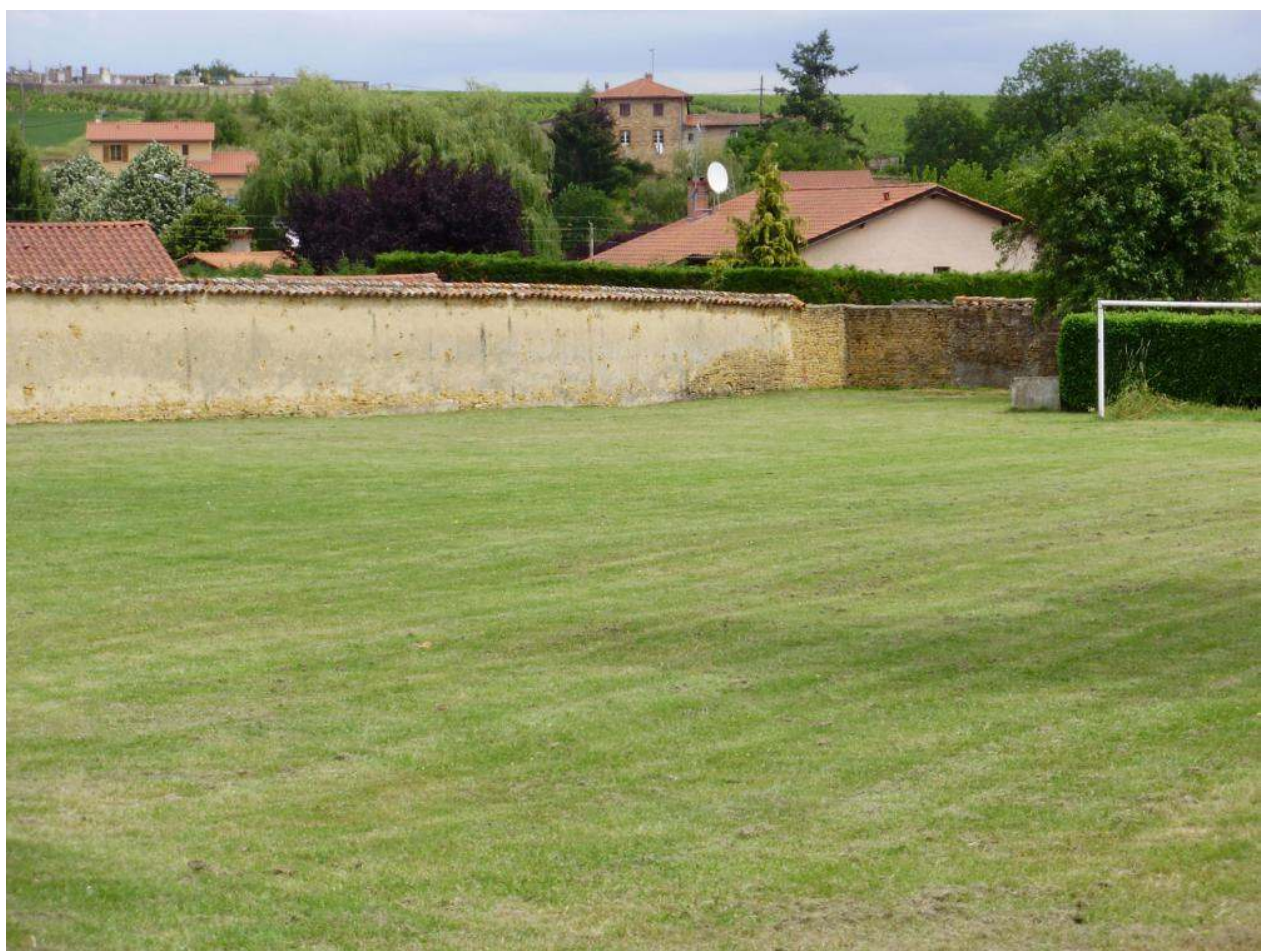

Fleur Guy, juin 2011

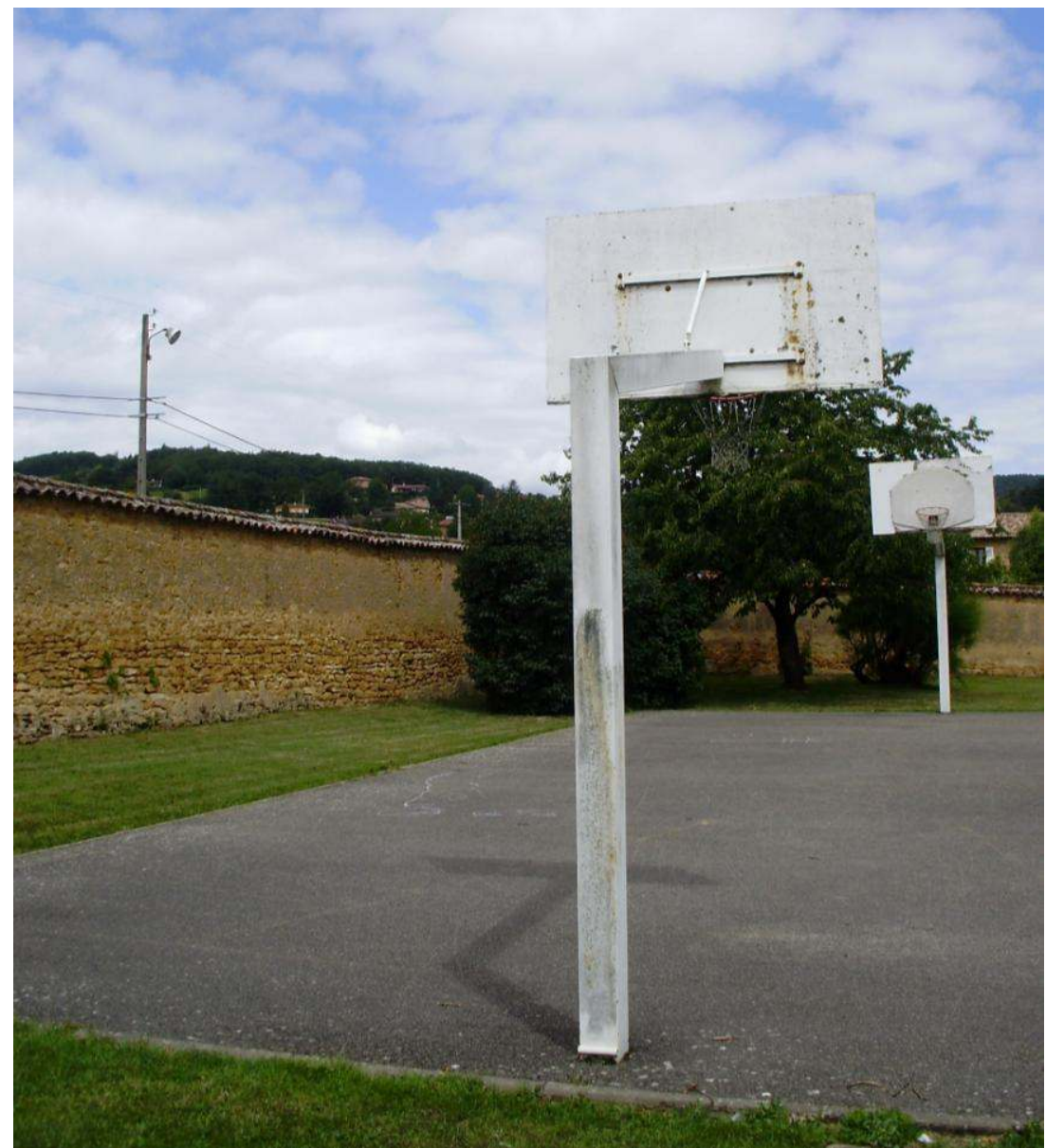

Fleur Guy, juin 2011 
Cette discontinuité entre l'établissement et son environnement invite à s'interroger sur la possibilité pour les mineurs accueillis d'établir des liens avec l'extérieur. La MECS "Les Alizés", située dans l'Ouest lyonnais, propose par exemple une prise en charge scolaire et médicale des enfants au sein même de l'établissement. La directrice dénonce le "côté asilaire et carcéral " de ce type d'établissements qui, bien qu'en évolution vers une prise en charge plus ouverte vers l'extérieur, peut parfois conduire au confinement des enfants. En effet, la scolarité et les loisirs sont souvent l'occasion de renouer des contacts entre le mineur et l'environnement extérieur et, à terme, de favoriser leur insertion sociale.

La question de la discontinuité entre les établissements et leur environnement se pose également dans le cas de structures dont la «fermeture " est au centre du projet. Issus de la loi Perben I (LOPJ), les CEF ont été perçus par les professionnels du domaine et certains chercheurs comme une entorse à la primauté de l'éducatif sur la sanction, principe fondateur de la juridiction spécialisée des mineurs instituée par l'ordonnance de 1945 (Goguel D'allondans, 2010 ; Mucchielli, 2005). Les CEF accueillent des jeunes en application d'un contrôle judiciaire, d'un sursis avec mise à l'épreuve, d'un placement extérieur dans le cadre d'un aménagement de peine ou à la suite d'une libération conditionnelle. Le CEF se caractérise par une "fermeture juridique ": "le non respect par le mineur des conditions du placement et des obligations fixées par la décision du magistrat pouvant entrainer sa détention » (DPJJ, 2010 : 10). Dans les faits, le CEF correspond souvent à la " dernière chance " avant l'incarcération et possède bien un caractère "fermé ". Pierre-Victor Tournier intègre ainsi les CEF à sa "mosaïque pénitentiaire ". Etablissant une "typologie des mesures et sanctions pénales", il note ainsi que " certaines personnes peuvent n'être pas sous écrou, tout en étant d'une certaine manière "enfermées " " (2006: 8). La proximité des établissements avec le milieu familial et social est utilisée comme un argument pour justifier l'augmentation de ce type d'établissements: "Si l'on veut que les centres soient proches de l'environnement social du jeune, il en faut au moins un par département, ce que la loi va permettre " (Roché, 2002 : 44). Cette réflexion sur la localisation des établissements gagnerait certainement à s'accompagner d'un questionnement autour des possibilités même de contact avec l'extérieur pour les mineurs placés. En effet, l'impératif du maintien des liens familiaux reste inscrit dans le cahier des charges des établissements mais doit s'articuler à la nécessaire " rupture avec leur environnement " pathogène " et maltraitant " et la mise en place " des cadres et modes de prise en charge des jeunes plus contraignants et plus contenants " (Darnaud, $2010: 62$ ).

\section{Mise à distance et/ou proximité : une articulation différenciée}

La mise en relation des différents types d'établissements, de publics et de l'articulation des objectifs de mise à distance et proximité présente tout d'abord certaines limites. Un même établissement peut en effet accueillir différents types de publics, alors même que les catégorisations utilisées peuvent être remises en cause, comme on l'a vu en première partie. Les établissements possédant une double habilitation, Justice et ASE, et ceux intervenant seulement dans le cadre d'un placement judiciaire peuvent cependant être différenciés. 
Figure 4 : L'articulation des objectifs de mise à distance et de proximité selon les types d'établissements

\begin{tabular}{|llccc|}
\hline Type d'établissement & Habilitation & $\begin{array}{c}\text { Distance spatiale } \\
\text { avec le domicile } \\
\text { familial }\end{array}$ & $\begin{array}{c}\text { Discontinuité avec } \\
\text { l'environnement } \\
\text { extérieur }\end{array}$ \\
\hline MECS & ASE/Justice & variable & variable \\
\hline CER & dans l'établissement & ASE/Justice & forte & forte \\
\cline { 2 - 5 } & séjour de rupture & ASE/Justice & très forte & faible \\
\hline CEF & Justice & forte & forte \\
\hline \hline EPM & Justice & faible & très forte \\
\hline
\end{tabular}

L'articulation de ces deux objectifs peut être analysée en évaluant la distance avec le domicile familial et la discontinuité avec l'environnement extérieur. Dans le cas des MECS, ces objectifs sont aléatoires, en lien avec la variabilité des établissements et des publics accueillis. En revanche, les CEF, CER et EPM se situent plus clairement dans une mise à distance, avec toutefois des modalités différentes. Le CER constitue un dispositif original, fondé sur l'éloignement du domicile familial et l'immersion dans un nouvel environnement, dans le cadre du séjour de rupture. Cette immersion doit cependant être nuancée puisque la prise en charge cherche à limiter les contacts avec les populations locales. Dans le cas de l'EPM et du CEF, la mise à distance apparaît comme un objectif prioritaire de la prise en charge de jeunes dans un cadre judiciaire pénal qui passe par une mise à distance géographique dans le cas du CEF et d'une discontinuité forte avec l'environnement extérieur dans le cas de l'EPM. La correspondance entre mise à distance et prise en charge judiciaire doit cependant être relativisée. Certaines MECS, dont les directeurs ont précisé qu'elles n'avaient pas vocation à accueillir de jeunes aux "profils délinquants ", répondent à la double caractéristique de la distance géographique avec le domicile familial et de la discontinuité avec l'environnement extérieur. La hiérarchisation des logiques de mise à distance et de proximité selon le type d'établissements et de publics nécessite ainsi une analyse plus fine, à l'échelle de l'établissement (cahiers des charges et projets d'établissements), mais aussi à l'échelle individuelle (ordonnance de placement et son application). Il est également important de considérer les différentes échelles spatiales et temporelles afin de relativiser cette première classification. 


\section{Conclusion}

La mesure de placement de mineurs hors de leur domicile familial et dans un établissement collectif permet de s'interroger sur la dimension spatiale d'un dispositif d'aide et d'action sociale. Au-delà de la dialectique entre éducation et sanction, cette prise en charge articule des objectifs qui peuvent apparaître contradictoires. En effet, la mise à distance sur laquelle s'est fondée la prise en charge de "l'enfance inadaptée " aux XVIII ${ }^{\text {ème }}$ et XIX ${ }^{\text {ème }}$ siècles doit aujourd'hui s'adapter à de nouveauX impératifs : maintien des liens familiaux du mineur placé mais également maintien de son lien avec l'environnement extérieur, notamment avec les structures liées à son insertion. Au cœur de cette dialectique entre mise à distance et proximité, la localisation des établissements est questionnée. La distance et l'accessibilité des établissements sont prises en compte de manière croissante par les professionnels de ces domaines. L'utilisation de l'espace géographique comme moyen d'action sur les mineurs en difficulté est plus ou moins affirmée, selon les types d'établissements et les publics accueillis. La mise à distance et la rupture sont par exemple au cœur de la prise en charge en CER et CEF. A une échelle plus fine, l'objectif de proximité avec l'environnement familial interroge les modalités de relation entre les mineurs placés en établissement et l'environnement extérieur, autant dans le cadre de structures dites "ouvertes", comme les MECS, que plus "fermées", comme les CEF. Se pose alors la question de la construction du rapport à l'espace pour les mineurs placés en établissement, plus particulièrement pour de longues durées. L'acquisition de compétences spatiales, entendues comme "les savoirs sur l'espace, la capacité à se projeter ailleurs, à la fois en terme de représentations (le champ des possibles) et de pratiques concrètes» (Ripoll, Veschambre, 2005: 478), considérées comme nécessaires à l'insertion sociale, telles que la mobilité (Bacqué, Fol, 2007), mériterait d'être interrogée dans ce contexte. 


\section{Bibliographie}

ARIES P. (1960), L'enfant et la vie familiale sous l'Ancien Régime, Paris, Plon, 502p.

BACQUE M-H., FOL S. (2007), "L'inégalité face à la mobilité: du constat à I'injonction ", Revue Suisse de Sociologie, 2007/33(1), pp. 89-104.

BORGETTO M., LAFORE R. (2009), Droit de l'aide et de l'action sociales, Paris, Editions Montchrestien, 740p.

BOURDIEU P. (1980), " La jeunesse n'est qu'un mot " in Questions de sociologie, Paris, Les Editions de Minuit, pp. 143-154.

BOURDIEU P. (1993), " Effets de lieu » in La misère du monde, Paris, Editions du Seuil, pp. 159-167.

BOURQUIN J. (2002), " Jeunes délinquants entre éducation et punition, le fantôme des maisons de redressement ", Le Monde diplomatique, 2002/579, p. 3.

BOURQUIN J. (2007), "Le mineur de justice, enfance coupable, enfance victime ", Revue d'histoire de l'enfance "irrégulière », Hors série 2007, pp. 129-140.

BOUQUET B., MADELIN B., NIVOLLE P. (2007), Territoires et action sociale, Paris, L'Harmattan, 343p.

CHOUFRI F. (2010), "La construction de la loi du 5 mars 2007. Pour une analyse sociopolitique des mutations de la Protection de l'enfance ", Sociétés et jeunesses en difficulté, 2010/9, http://sejed.revues.org/index6715.html, Consulté le 11 janvier 2011.

Comité interministériel de l'évaluation des politiques publiques, Commissariat général du Plan (1993), L'insertion des adolescents en difficulté, Rapport d'évaluation, Paris, La Documentation française, $374 \mathrm{p}$.

CRESSWELL T. (1996), In place/out of place: geography, ideology, and transgression, London, University of Minnesota Press, 201p.

DARNAUD Y. (2010), " CER et CEF : un travail possible ", Contenir ou Enfermer, V.S.T. Revue du champ social et de la santé mentale, 2010/108, pp. 61-64.

Direction de la Protection Judicaire de la Jeunesse (2010), Guide des assesseurs du tribunal pour enfants, 20p.

DUGAT G., LAMARQUE J. (1850), Des colonies agricoles établies en France en faveur des jeunes détenus, enfants trouvés, pauvres, orphelins ou abandonnés, précis historique et statistique, Paris, Imprimerie de Rignoux, 148p.

GACHELIN M. (2007), « Les jeunes et la mobilité », EMPAN, 2007/3(67), pp. 60-64.

JABLONKA I. (2010), Les enfants de la République. L'intégration des jeunes de 1789 à nos jours, Paris, Editions du seuil, 348p. 
JAMET L. (2010), "Les mesures de placement de mineurs "délinquants »: entre logiques institutionnelles et stigmatisation du public ", Sociétés et jeunesses en difficulté, http://sejed.revues.org/index6689.html, Consulté le 11 janvier 2011.

GOGUEL D'ALLONDANS T. (2010), "Les nouveaux visages de l'éducation renforcée: CEF, EPM, EPIDE », Contenir ou Enfermer, V.S.T. Revue du champ social et de la santé mentale, 2010/108, pp. 54-60.

HOLLOWAY S., VALENTINE G. (2000), Children's geographies: playing, living, learning, London, New York, Routledge, 275p.

LAPEYRONNIE D. (dir) (2003), Des Opérations Prévention Eté à Ville Vie Vacances 1982 2002, Saint-Denis, Editions de la DIV, 218p.

LUSSAULT M., STOCK M. (2010), " " Doing with space ": towards a pragmatics of space ", Social Geography, http://www.soc-geogr.net/5/11/2010/sg-5-11-2010.html, Consulté le 19 avril 2011.

MILHAUD O. (2009), "Séparer et punir. Les prisons françaises: mise à distance et punition par l'espace ", Thèse de doctorat en géographie sous la direction de G. Di Méo, Université Bordeaux 3, 368p.

MORELLE M. (2010), "La rue, un espace à négocier Yaoundé (Cameroun) et Antananarivo (Madagascar) ", in Danic I., David D., Depeau S. (dir.), Enfants et jeunes dans les espaces du quotidien, Rennes, Presses Universitaires de Rennes, pp. 233-246.

MUCCHIELLI L. (2005), "Les "Centres Educatifs Fermés » : rupture ou continuité dans le traitement des mineurs délinquants ? ", Revue d'histoire de l'enfance "irrégulière ", http://rhei.revues.org/index1038.htlm, Consulté le 18 novembre 2010.

PEDRON P. (2005), Droit et pratique de la Protection Judicaire de la Jeunesse, Mineurs en danger, mineurs délinquants, Paris, Gualino Editeur, 416p.

POUPEAU F., TISSOT S. (2004), "La spatialisation des problèmes sociaux ", Actes de la recherches en sciences sociales, http://www.cairn.info/revue-actes-de-la-rechercheen-sciences-sociales-2005-4-page-4.htm, consulté le 19 novembre 2010.

RIPOLL F. (2006), " Du " rôle de l'espace » aux théories de " l'acteur » (aller-retour) La géographie à l'épreuve des mouvements sociaux ", in Séchet R., Veschambre V., Penser et faire la géographie sociale, Contributions à une épistémologie de la géographie sociale, Rennes, Presses Universitaires de Rennes, pp. 192-210.

RIPOLL F., TISSOT S. (2010), "La dimension spatiale des ressources sociales ", Regards sociologiques, 2010/40, pp. 5-7.

RIPOLL F., VESCHAMBRE V. (2005), "Sur la dimension spatiale des inégalités: contribution aux débats sur la " mobilité et le capital spatial " ", in Arlaud S., Jean Y., ROYOUX D., Rural-urbain, Nouveaux liens, nouvelles frontières, Rennes, Presses universitaires de Rennes, pp. 467-483. 
ROCHE S. (2002), "Les centres éducatifs fermés : un procès excessif ", in " Sécurité et justice » Regards sur l'actualité, 2002/284, Paris, La documentation française, pp. 4145.

Sénat (2002), Rapport $n^{\circ} 340$ de la commission d'enquête sur la délinquance des mineurs créée en vertu d'une résolution adoptée par le Sénat le 12 février 2002 (prés. SCHOSTECK J-P), dépôt publié au JO du 27 juin 2002.

SICOT F., MAIGNE N. (2005), " Les CER, un placement (pénal) parmi d'autres ? ", EMPAN, 2005/3(59), pp. 91-104.

TESSIER S. (dir.) (1995), L'enfant des rues et son univers. Ville, socialisation et marginalité, Paris, SYROS, 228p.

TISSOT S. (2007), L'Etat et les quartiers, genèse d'une catégorie de l'action publique, Paris, Editions du Seuil, 300p.

TOURNIER P-V. (2006), " Détention, alternatives à la détention », Champ pénal / Penal field, nouvelle revue internationale de criminologie, http://champpenal.revues.org/567, Consulté le 18 octobre 2010.

VESCHAMBRE V., (2006), "Penser l'espace comme dimension de la société ", in Séchet R., VESCHAMBRE V., Penser et faire la géographie sociale, Contributions à une épistémologie de la géographie sociale, Rennes, Presses Universitaires de Rennes, pp. 221-227.

WACQUANT L. (2001), "Symbiose fatale Quand ghetto et prison se ressemblent et s'assemblent», Actes de la recherche en sciences sociales, 2001/139, pp. 31-52. 